\title{
Rotational motions in seismology: theory, observation, simulation
}

\author{
A. Cochard * H. Igel * B. Schuberth * \\ cochard@geophysik.uni-muenchen.de

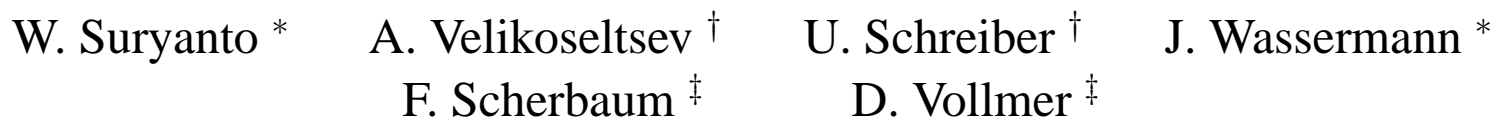

October 17, 2005

Keywords:

rotational motions, wave propagation, surface waves, seismic instrumentation

\section{Introduction}

The rotational part of earthquake-induced ground motion has basically been ignored in the past decades, compared to the substantial research in observing, processing and inverting translational ground motions, even though there are theoretical considerations that suggest that the observation of such motions may indeed be useful and provide additional information. In the past years, interest in this potentially new observable for seismology has risen, primarily because -with modern acquisition technology such as fiber-optical or ring laser gyros- rotational motions were actually observed, the resolution is steadily increasing, and the observations are becoming consistent with collocated recordings of translational ground motions. Even though the real benefit to Earth sciences is still under investigation, recent results suggest that collocated measurements of rotations and translations may allow the estimation of wavefield properties (such as phase velocities, direction of propagation) that otherwise can only be determined through array measurements or additional strain observations. In this paper we focus on studies of the vertical rotational component (twist, spin, or rotation around a vertical axis) and review recent results on the fundamental concepts that are necessary to understand the current broadband observations of a wide distance and magnitude range, and show that the classical theory of

\footnotetext{
${ }^{1}$ Department of Earth and Environmental Sciences, Ludwig-Maximilians-Universität, Theresienstr. 41, 80333 München, Germany

${ }^{2}$ Forschungseinrichtung Satellitengeodäsie, Technical University of Munich, Fundamentalstation Wettzell, Sackenriederstr. 25, D-93444 Kötzting, Germany

${ }^{3}$ Institut für Geowissenschaften, Universität Potsdam, Karl-Liebknecht-Str. 24/25, 14476 Golm, Germany
} 
linear elasticity is sufficient to explain these observations. In addition to direct measurements of rotational motions using ring laser technology, we describe the method to derive rotational motions from seismic arrays and present some initial results. Sophisticated 3D modelling of the rotational ground motions of teleseismic events illustrate the accuracy with which observed horizontal phase velocities match with theoretical predictions, even though the precise waveforms are quite different due to inaccuracies in crustal models or kinematic rupture properties. This may have implications for sparse networks or situations where extremely few or even single-station observations are taken (e.g., in remote areas or planetary seismology).

To fully characterize the motion of a deformable body at a given point in the context of infinitesimal deformation, one needs three components of translation, six components of strain, and three components of rotation, a vectorial quantity. Rotational motions induced by seismic waves have been essentially ignored for a long time, first because rotational effects were thought to be small (Bouchon and Aki 1982), and second because sensitive measuring devices were not available. Indeed, Aki and Richards (1980, p. 489) point out that "the state-of-the-art sensitivity of the general rotation sensor is not yet enough for a useful geophysical application."

However, there have been many reports of rotational effects associated with earthquakes (like twisting of tombstones, or statues). It is certainly possible that some of these effects are due to the asymmetry of the construction. Indeed, as is well known, when the center of mass is not located at the geometric center, a mere translation may induce a local rotation of that structure. However, some field evidences suggest that it is at least not always the case (Galitzin 1914, p. 172). The rotational angles calculated by Bouchon and Aki (1982) for realistic cases of earthquake scenarios (about $10^{-4}$ radians) seem indeed too small to be responsible for damages, except, maybe for the case of long structures. However, as, roughly speaking, rotations are proportional to displacement divided by the phase velocity (see section 2.3), when the wave velocity becomes smaller, rotations become comparatively larger. This happens in soft or unconsolidated sedimentary and/or fluid-infiltrated porous media, where wave speeds might be as low as about $50 \mathrm{~m} / \mathrm{s}$, hence about a factor of 50 smaller than usual. Thus, it is not implausible that, near seismic sources -where rotations and strains become relatively large even in normal media-, rotations and strains become really large and be responsible for the above mentioned damages (there is also growing seismological evidence that rotational amplitudes have been underestimated (Castellani and Zembaty 1996)). Obviously, in such a situation, the assumption of infinitesimal deformation would then break and the theory of finite deformations would be necessary.

As an attempt to measure rotational motions with high sensitivity, in the past years ring laser gyroscopes were developed, primarily to observe variations in Earth's absolute rotation rate with high precision (Stedman et al. 1995; Stedman 1997). One of these instruments -located near Christchurch, New Zealand- recorded seismically induced signals of ground rotation rate for several large earthquakes (McLeod et al. 1998; Pancha et al. 2000). These observations gave evidence that the optical sensors indeed provide sufficient accuracy to record seismic rotations. However, they were not fully consistent in phase and amplitude with translational motions recorded with collocated seismometers, the limited consistency being obtained only in a narrow 
frequency band. Earlier attempts to observe ground rotations with other devices (e.g., solid state rotational velocity sensors, fiber-optical gyros) were limited to large signals close to artificial or earthquake sources (Nigbor 1994; Takeo 1998) and did not lead so far to an instrument of general interest. This explains that Aki and Richards (2002, p. 608) note that "as of this writing seismology still awaits a suitable instrument for making such measurements." However, the subsequent development of ring laser technology indicates that a significant part of the gap has recently been filled, as demonstrated by Schreiber et al. (2003b) and Igel et al. (2005b) and exemplified later in this paper. Rotations can also be determined with array measurements, but with important limitations; see section 3.1 and Suryanto et al. (2005).

The recording of even small, non potentially damaging, rotational motions is expected to be very useful. First, translation recordings are polluted by rotations. There is a purely geometrical effect (the reference axis of the seismometer is rotated), introducing a cosine factor, hence negligible for very small deformations, but which could become significant in case of very strong ground motion. More importantly, there is also an inertial contribution. It is well known that surface tilt (horizontal rotation - see section 2.2) induces a translational signal (Aki and Richards 2002, p. 604). A similar effect exists for vertical rotation (Trifunac and Todorovska 2001). Measuring the three components of rotation allows in principle to correct for these effects. Second, as the measure of rotation provides additional information, it helps constraining physical models. For example, the measure of vertical rotation and horizontal acceleration allows the estimation of the local Love wave phase velocity and of its propagation direction (see section 2.3 and Igel et al. (2005a)). It is also expected (Takeo and Ito 1997) that the measure of rotations will allow to better constrain earthquake rupture histories.

The above discussion has been deliberately restricted to classical elasticity, for which the stress and strain tensors are symmetric. In some extreme cases (e.g., large stress gradient) near crack tips or in granular materials, the material can no longer be treated as a continuum. A continuous formalism may still be used for an effective medium in which additional -intrinsic- rotations (i.e., not related to displacement) may exist. In such Cosserat/micropolar media, the stress and strain tensors are no longer symmetric. See, e.g., Dyszlewicz (2004); Nowacki (1986); Maugin (1998); Lakes (1995); Majewski (2001); Teisseyre et al. (2003), and other papers in this issue. In the following of this paper we remain in the framework of classical elasticity.

\section{Fundamental theory}

In the framework of classical elasticity, and further assuming infinitesimal deformations, displacement of a point $\mathbf{x}$ is related to that of a neighboring point $\mathbf{x}+\delta \mathbf{x}$ by (e.g., Aki and Richards 2002, p. 13)

$$
\begin{aligned}
\mathbf{u}(\mathbf{x}+\delta \mathbf{x}) & =\mathbf{u}(\mathbf{x})+\mathbf{G} \delta \mathbf{x} \\
& =\mathbf{u}(\mathbf{x})+\boldsymbol{\varepsilon} \delta \mathbf{x}+\boldsymbol{\Omega} \delta \mathbf{x} \\
& =\mathbf{u}(\mathbf{x})+\boldsymbol{\varepsilon} \delta \mathbf{x}+\boldsymbol{\omega} \times \delta \mathbf{x}
\end{aligned}
$$




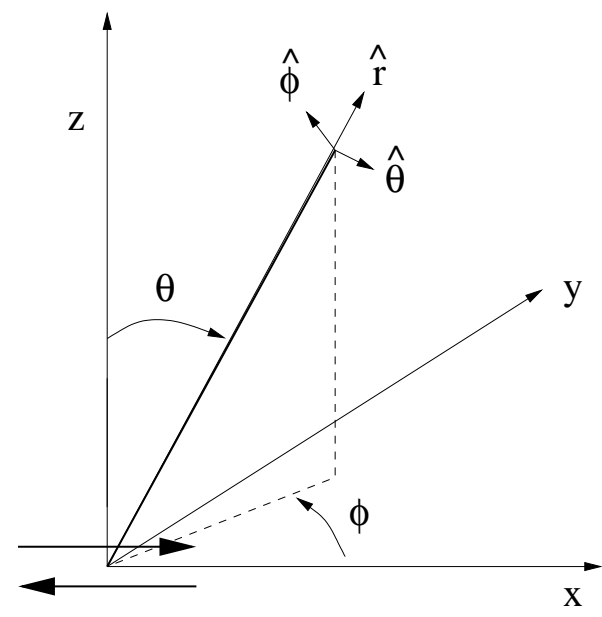

Figure 1: Geometry and notations for the double couple expressions.

where $\mathbf{G}, \varepsilon, \Omega$ are the gradient, strain, and rotation second order tensors, respectively, and

$$
\boldsymbol{\omega}=\frac{1}{2} \nabla \times \mathbf{u}(\mathbf{x})
$$

is a (pseudo-) vector which does not enter Hooke's law and represents the angle of rigid rotation generated by the disturbance. This illustrates that one needs three components of translation, six components of strain and three components of rotation to fully characterize the change in the medium around point $\mathbf{x}$.

\subsection{Rotations and strain due to a double couple point source}

Aki and Richards (2002, figure 4.4 and eqs. (4.32) and (4.33)) give the expression for the displacement $\mathbf{u}(\mathbf{x})$ generated by a point source shear dislocation (double couple) in an infinite, homogeneous, isotropic medium. The dislocation is in the $x-y$ plane with slip along $x$ (figure 1).

Following the same notations, we can then determine the rotation as

$$
\begin{aligned}
\boldsymbol{\omega}(\mathbf{x}, t) & =\frac{1}{2} \nabla \times \mathbf{u}(\mathbf{x}, t) \\
& =\frac{-\mathbf{A}^{R}}{8 \pi \rho}\left[\frac{3}{\beta^{2} r^{3}} M_{0}\left(t-\frac{r}{\beta}\right)+\frac{3}{\beta^{3} r^{2}} \dot{M}_{0}\left(t-\frac{r}{\beta}\right)+\frac{1}{\beta^{4} r} \ddot{M}_{0}\left(t-\frac{r}{\beta}\right)\right]
\end{aligned}
$$

where

$$
\mathbf{A}^{R}=\cos \theta \sin \phi \hat{\boldsymbol{\theta}}+\cos \phi \cos 2 \theta \hat{\boldsymbol{\phi}}
$$

is the radiation pattern of the three components of rotation (the radial component being zero). Likewise, the divergence (or trace of the strain tensor, representing volumetric change) is given 

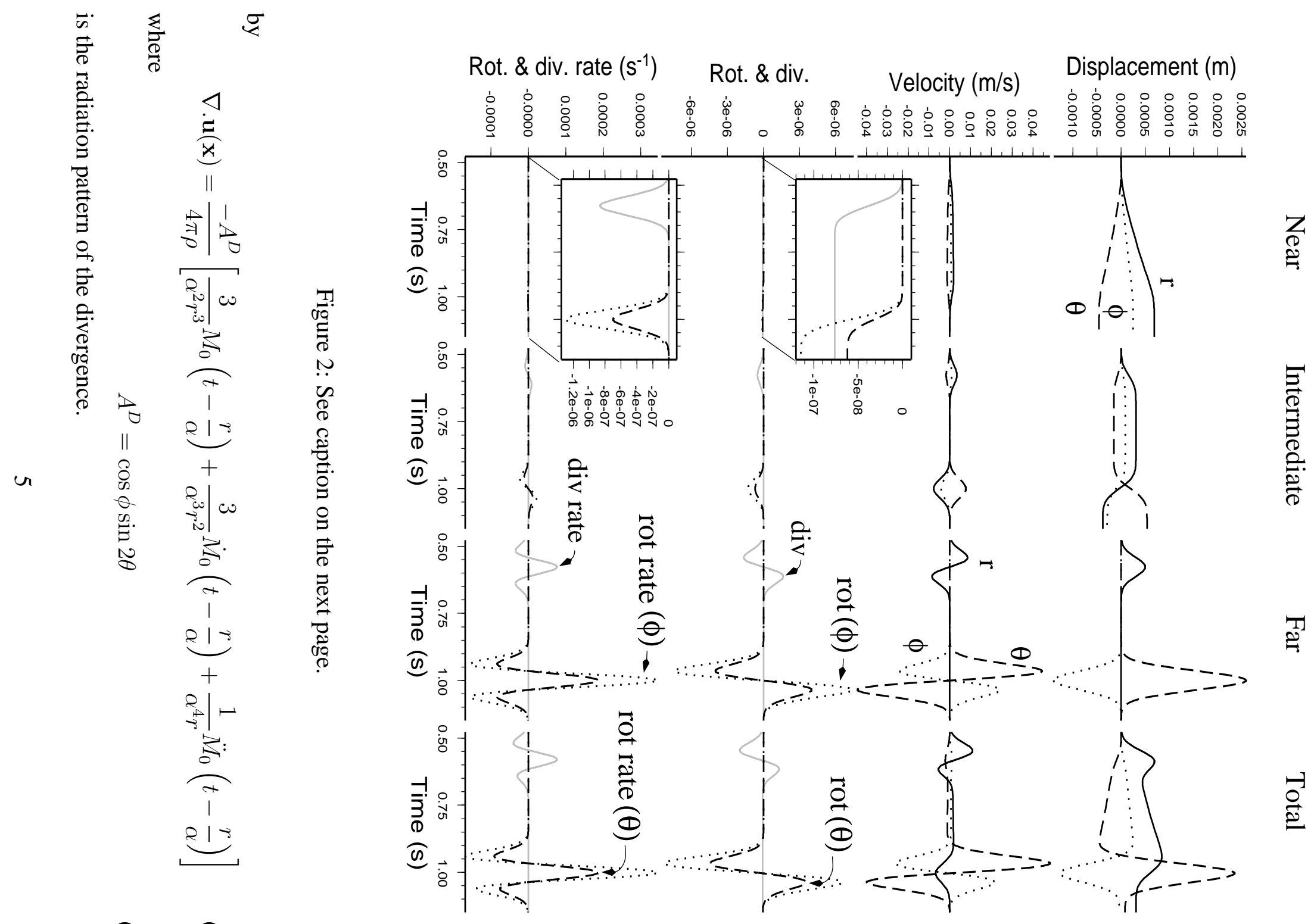

a) 
Figure 2: (continued) From top to bottom: Displacement, velocity, divergence and rotation, divergence and rotation rates, as a function of time at a given point due to a double couple in an infinite, homogeneous, isotropic medium. The dislocation is in the $x-y$ plane with slip along $x$, as in Aki and Richards (2002, figure 4.4). The observation point is defined as $(r, \theta, \phi)=(3 \mathrm{~km}, \pi / 8, \pi / 8)$ in spherical coordinates. The moment is $10^{15} \mathrm{~N} \cdot \mathrm{m}(M \approx 4)$ and the source time function rate is a Gaussian of the form $\exp \left(-(t / T)^{2}\right)$ with $T=0.05 \mathrm{~s}$. The density of the medium is $\rho=2800 \mathrm{~kg} / \mathrm{m}^{3}$, the $S$ and $P$ wave velocities are $\beta=3 \mathrm{~km} / \mathrm{s}$ and $\alpha=\sqrt{3} \beta$. Concerning displacement and velocity, the usual terminology is used for the near, intermediate, and far fields, but not concerning divergence and rotation (and their rates) (see text). The radial components (along $\hat{r}$ ) are plotted black, the $\hat{\theta}$ components, dashed, and the $\hat{\phi}$ components, dotted; the divergence and divergence rate evolutions are plotted gray.

Figure 2 shows displacement, velocity, divergence and rotation, and divergence and rotation rates, as a function of time at a given point (see caption for details).

Intuitive from eq. (1) -since the gradient tensor is merely split into symmetric and antisymmetric tensors-, and apparent from the figure, is that rotations and strains are of the same order of magnitude.

Not surprisingly, rotations are zero at the $\mathrm{P}$ wave front, and only start at the $\mathrm{S}$ wave arrival (and have no radial component), even in the displacement near field region (this is also true for, e.g., single forces or single couples). This could however appear strange at first sight, as, e.g., the rotation associated with the far field $\mathrm{P}$ term is not zero. This is obvious when looking at its radiation pattern (figure 4.5 (a) of Aki and Richards 2002) and is in fact given by $-\mathbf{A}^{R} \dot{M}_{0}(t-r / \alpha) /\left(4 \pi \rho \alpha^{3} r^{2}\right)$. It turns out that this contribution cancels out with part of the curl of the intermediate field at the $\mathrm{P}$ front, the other part canceling in turn with the near field contribution. (Nevertheless, rotations may not be obtained by taking the curl of only the transverse displacement terms in eq. (4.32) of Aki and Richards (2002).) Analogous comments may be made for strains, which are active around the $\mathrm{P}$ front only. Therefore, the usual classification for displacement in near, intermediate, and far field terms cannot be applied to rotations and strains. As rotations and strains comprise terms proportional to $1 / r, 1 / r^{2}, 1 / r^{3}$, it is convenient to refer to them as far, intermediate, and near field terms, respectively, which has been done in figure 2. However, this could be misleading, since, if the far and intermediate displacement fields also decrease as $1 / r$ and $1 / r^{2}$, respectively, the near field displacement term, proportional to $\left(1 / r^{4}\right) \int_{r / \alpha}^{r / \beta} \tau M_{0}(t-\tau) d \tau$ essentially decreases as $1 / r^{2}$, not $1 / r^{3}$.

We conclude the section by noting that the rotation radiation pattern $\mathrm{A}^{R}$ can be calculated from the transverse (or $\mathbf{S}$ ) far field radiation pattern $\mathbf{A}^{F S}=\cos 2 \theta \cos \phi \hat{\boldsymbol{\theta}}-\cos \theta \sin \phi \hat{\boldsymbol{\phi}}$ (eq. (4.33) of Aki and Richards 2002, p. 78) as $\nabla \times \mathbf{A}^{F S}=\mathbf{A}^{R} / r$; thus, $\mathbf{A}^{F S}$ and $\mathbf{A}^{R}$ are orthogonal. Similarly, $A^{D}$ is related to the radial (or P) far field radiation pattern $\mathbf{A}^{F P}=\mathbf{A}^{D} \mathbf{r}$. 


\subsection{Effect of free surface - rotation and tilt}

Assuming the surface corresponds to the $x-y$ plane, the zero traction boundary condition at the free surface implies that $\sigma_{i 3}=0(i=x, y, z)$. Direct application of Hooke's law $\sigma_{i j}=$ $\lambda \epsilon_{k k} \delta_{i j}+2 \mu \epsilon_{i j}$ in a homogeneous, isotropic medium leads to

$$
\frac{\partial u_{x}}{\partial x_{z}}=-\frac{\partial u_{z}}{\partial x_{x}} \quad ; \quad \frac{\partial u_{y}}{\partial x_{z}}=-\frac{\partial u_{z}}{\partial x_{y}} \quad ; \quad \frac{\partial u_{z}}{\partial x_{z}}=-\frac{\lambda}{\lambda+2 \mu}\left(\frac{\partial u_{x}}{\partial x_{x}}+\frac{\partial u_{y}}{\partial x_{y}}\right) .
$$

It then directly follows (eq. (2)) that

$$
\omega_{x}=\frac{\partial u_{z}}{\partial x_{y}} \quad ; \quad \omega_{y}=-\frac{\partial u_{z}}{\partial x_{x}},
$$

hence that at the Earth's surface, horizontal rotation corresponds to tilt.

As a consequence, $\mathrm{P}$ wave generate horizontal rotation at the surface whereas they are irrotational in the bulk. There is an additional contribution to horizontal rotation due to P-to-SV converted waves at the surface (also due to the free surface boundary condition). It is still an open question what technology is best suitable for the various components of rotation. For the vertical component, the results presented here and in other studies suggest that the optical devices are the way to go. Those optical devices could be advantageous compared to classical tiltmeters for the horizontal components as well (regardless of meteorological characteristics -accuracy, sensitivity, etc.- which remain to be assessed): indeed, classical tiltmeters measure a change of angle with respect to the local vertical, determined by gravity; thus they cannot discriminate between true rotation and a pure change in the local gravity (e.g., due to mass redistribution).

\subsection{Comparison of rotation and translations}

Obviously, if we know, or assume we know, the full expression for displacement $\mathbf{u}$, we can determine rotation $\boldsymbol{\omega}$ from the measurement of $\mathbf{u}$ (eq. (2)) by differentiating. If we do not fully know $\mathbf{u}$, the measurement of $\boldsymbol{\omega}$ brings in additional information.

First, consider the case of a transversely polarized plane wave with displacement $\mathbf{u}(x, y, z, t)=\left(0, u_{y}(t-x / c), 0\right)$ propagating in the $x$ direction, with $c$ being the horizontal phase velocity. Rotation is thus given as $\boldsymbol{\omega}(x, y, z, t)=(1 / 2) \nabla \times \mathbf{u}(x, y, z, t)=$ $\left(0,0,-\dot{u}_{y}(t-x / c) /(2 c)\right)$. Thus we have

$$
\omega_{z}(x, y, z, t)=-\frac{\dot{u}_{y}(x, y, z, t)}{2 c} .
$$

Surface waves are composed of both Love waves, with horizontal transverse polarization, and Rayleigh waves, with displacement in a vertical plane assuming elastic isotropy. So Rayleigh waves do not generate a vertical component of rotation. Thus, for a given earthquake for which the distance to the station is large enough compared with the source dimension and 
the wavelength of interest so that the plane wave assumption is valid, from the measurement of rotation and displacement of a surface wave train one can in principle infer both the phase velocity and the direction of the incoming wave train. In practice, we have so far dealt only with earthquakes of known location, and have assumed that the surface trains were traveling along the great circle, thus providing the direction (Igel et al. 2005b, and section 4 below). There are now observations of ground rotations of several dozens of earthquakes with a wide magnitude and epicentral distance range. The accuracy of the phase velocity estimates is currently under investigation by comparison with complete wave simulations (see below), and array-derived phase velocities (Suryanto et al. 2005) as well as theoretical predictions of Love-wave dispersion for local Earth models.

\section{Rotational measurements}

Rotational ground motions can be observed by (1) finite-differencing of seismic array data or (2) directly (ring laser, fiber-optical or mechanical gyros). In this section we briefly review both approaches and give relevant references.

\subsection{Array derived rotations}

One way of estimating the components of the deformation gradient $\mathbf{G}$, hence of computing strains and rotations, is through array measurements (e.g., Spudich et al. 1995; Bodin et al. 1997; Huang 2003). Using the first of eqs. (1) with a non infinitesimal quantity $\mathbf{R}$ as the position vector between two points in place of $\delta \mathbf{x}$, we get $\mathbf{d}=\mathbf{u}(\mathbf{x}+\mathbf{R})-\mathbf{u}(\mathbf{x})=\mathbf{G}^{F D} \mathbf{R}$, where $\mathbf{G}^{F D}$ is a first order approximation of $\mathbf{G}$, i.e., exact if $\mathbf{u}$ varies linearly between $\mathbf{x}$ and $\mathbf{x}+\mathbf{R}$. Using three non aligned points, one gets two such vectorial equations (six equations in Cartesian coordinates) with a common point, relating, say, $\mathbf{d}_{1}$ and $\mathbf{d}_{2}$ to $\mathbf{R}_{1}$ and $\mathbf{R}_{2}$, and one can then solve for the six approximations of the partial derivatives in the plane defined by the three points. Specifically, if we measure the ground displacement at three stations on the surface, whose relative positions are known, we can determine (without using the free surface boundary condition) estimations of $\partial u_{x} / \partial x, \partial u_{x} / \partial y, \partial u_{y} / \partial x, \partial u_{y} / \partial y, \partial u_{z} / \partial x, \partial u_{z} / \partial y$. This is what is commonly used in finite element methods to calculate the spatial derivatives in triangular cells (e.g., Cook 1974); for example, one gets $\partial u_{x} / \partial y=\left(d_{2 x} R_{1 x}-d_{1 x} R_{2 x}\right) /(2 S)$, where $S$ is the surface of the triangle.

In particular, we can determine an estimation of the vertical component of rotation

$$
\omega_{z}=\frac{1}{2}\left(\frac{\partial u_{x}}{\partial y}-\frac{\partial u_{y}}{\partial x}\right)
$$

When more than three stations are used, the equations $\mathbf{d}_{i}=\mathbf{G}^{F D} \mathbf{R}_{i}$ (where $i+1$ is the number of stations) can be solved by using a least square procedure (e.g., Spudich et al. 1995). This is how we proceed in 4.2 . 
Note that the rotation-induced translational signals, as explained in the introduction, affect the array-derived rotation calculations as described just above. It should be possible to devise an iterative procedure to correct for this effect, but this has not been attempted in this paper.

Using the free surface boundary condition, one can also estimate the horizontal components of rotation, and, if further assuming the quantity $\lambda /(\lambda+2 \mu)$ known, $\partial u_{z} / \partial z$ (cf. 2.2). Note that $\omega_{z}$ cannot be determined by means of tiltmeters or strainmeters. Array-derived rotation are subjected to an important limitation. The instruments should be as close as possible for the finite difference approximations to be as close as possible to the true gradients. However, for a given instrumental noise level, the uncertainty in the gradients will be larger if the instruments are closer; see Suryanto et al. (2005) for a more in-depth discussion of noise contribution. Finally, the assumption of linearity of the displacement will be greatly affected by site effects at the stations.

\subsection{Ring laser rotational measurements}

In 1913, G. Sagnac showed that two counter-propagating light beams, steered around a closed area $A$ inside an interferometer, generated a phase shift when the instrument was rotating at an angular frequency $\dot{\omega}$ with respect to an inertial frame. In our active square laser cavities, lasing at a single mode per sense of rotation is achieved when an integral number of wavelengths circumscribe the ring perimeter. Since the path length is slightly different for the co-rotating and counter-rotating laser beams, we obtain a beat frequency $\Delta \nu$ instead of the phase shift. This has the advantage that, technically, a frequency can be measured much more precisely than a phase shift. With $P$ the perimeter of our instrument, the Sagnac equation for an active ring laser cavity reads

$$
\Delta \nu=\frac{4 A \mathbf{n} \cdot \dot{\omega}}{\lambda P}
$$

where $\lambda$ is the rest wavelength of the laser and $\mathbf{n}$ is the unit vector perpendicular to the ring laser area. Inherently, this equation has three contributions which reflect upon the beat frequency $\Delta \nu$. Variations of the scale factor $(4 A / \lambda P)$ have to be avoided by making the instrument mechanically as rigid and stable as possible. Changes in orientation enter the beat frequency via the inner product. Finally, variations in $\dot{\omega}$ are representing the most dominant contribution to changes in $\Delta \nu$ and are the focus of our seismic studies here. Technically, our rings are realized as high $Q$ Helium/Neon lasers, operated close to laser threshold in order to satisfy the single mode condition (Stedman 1997). The body is made from Zerodur, a glass ceramic with extremely low thermal expansion, to ensure the required scaling factor stability. Mirrors with a total loss of around $15 \mathrm{ppm}$ each are used to minimize errors arising from coupling between the two counter-rotating beams through backscattered light. The larger a ring laser can be made, the higher is the sensitivity to rotation (Schreiber et al. 2003c).

All ring lasers are rigidly attached to bedrock and very small changes of orientation caused by Earth tides, ocean loading (Schreiber et al. 2003a) and in particular diurnal polar motion (Schreiber et al. 2004) were successfully detected. Ring lasers have two major advantages for 

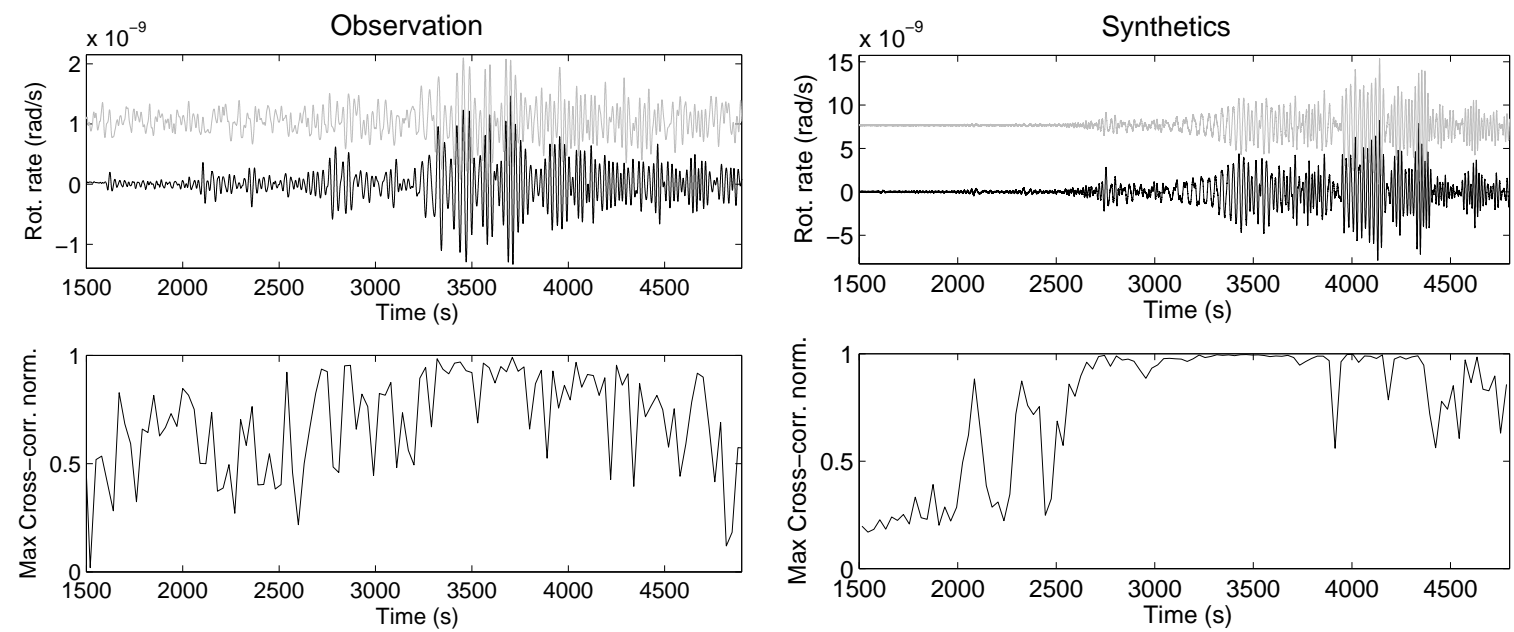

Figure 3: Comparison of correlation coefficients, obtained with a 30-second sliding window, of acceleration and rotation rate for the M7.3 Irian Jaya (Indonesia) earthquake of Feb. 07, 2004. Left: observed; right: synthetics. Top: scaled (see text) transverse acceleration (black) and rotation rate (gray - shifted for legibility).

applications in seismic studies. They are measuring absolute rotation with respect to the local universe and they do not depend on accelerated masses. In particular this last property ensures an extremely wide dynamic range of operation, reaching from a few $\mu \mathrm{Hz}$ for geophysical signals up to more than $10 \mathrm{~Hz}$ as obtained from regional earthquakes.

\section{Observations and simulations of rotational motions}

In this section we shall present direct (ring laser) and array-derived rotations and compare with observations of transverse acceleration and theoretical rotation rate calculated for a 3D Earth model.

\subsection{Ring laser observation of rotational motions, comparison with simu- lations}

In the last years the capabilities of simulating the propagation of seismic waves resulting from earthquakes on a global scale have improved significantly. This is not only due to the still increasing computer resources, but also due to improved and highly sophisticated numerical methods. The "spectral element method" (Komatitsch 1997; Komatitsch and Tromp 2002a,b) for example allows very accurate modeling of elastic waves in three-dimensional model structures of the Earth's interior, additionally incorporating various effects, such as topography/bathymetry, anelasticity, anisotropy, ocean loading and rotation as well as gravitation of the Earth. The 
method proved to give very good results in terms of fit to observed data when using current 3D tomographic earth models (Komatitsch et al. 2003; Igel et al. 2005b; Schuberth et al. 2005). For this study, we used a modified version of the spectral element program SPECFEM3D, in which we implemented the calculation and output of rotational ground motions for comparison with the ring laser data from Wettzell, Germany. The actual computation of the rotations in the code is straightforward, the spatial derivatives of the displacement $\partial u_{x} / \partial x$ etc. being already available as they are needed for the calculation of the translational motions. Thus we can easily obtain the rotational motion as the curl of the displacement field (eq. (2)).

For this study, simulations were carried out using a finite source model of the M7.3 Irian Jaya earthquake in Indonesia on Feb. 07, 2004 (Ji et al. 2002; Chen 2004). Most of the available options described above were incorporated in the model, thus including 3D models for the crust and the mantle (crust2.0 and S20RTS respectively), attenuation, transverse isotropy, topography/bathymetry, ocean loading as well as gravity and rotation of the Earth. The resolution of the model allowed for numerical seismograms accurate down to periods of 20 seconds.

As shown in 2.3, the rate of rotation and the transverse component of acceleration at the same location should be in phase for an incoming plane wave. Furthermore, when assuming a horizontally propagating wave, one can estimate the phase velocity. The results of the simulation demonstrate this in a very clear way, as the seismograms are inherently noise free. Figure 3 shows the comparison of observed rotation rate (gray) and transverse acceleration (black) for the M7.3 Irian Jaya earthquake of Feb. 07, 2004. The real data (top left) were filtered between 1000- and 20-second periods, that lower limit being controlled by the accuracy of the synthetic data. The amplitudes of acceleration were scaled by an arbitrary phase velocity of $3000 \mathrm{~m} / \mathrm{s}$. Shown in the lower left part of the figure is the correlation coefficient of a 30-second sliding window. Despite the relatively low signal-to-noise ratio in the ring laser data, the normalized correlation coefficient reaches values close to one (perfect match) for most of the surface wave train between $3200 \mathrm{~s}$ and $4200 \mathrm{~s}$. The right part of the figure shows the same for the modeled data which is inherently noise free. The correlation is higher for the simulated data, but showing a similar behavior for the body and surface wave parts of the seismogram. The synthetic data do not fit exactly the observed data, but show some strong similarities in the surface waves, where the signal to noise ratio (especially for the ring laser) gets better. The difference between synthetic and real data may either be due to an inaccurate model of the rupture process and/or to incorrect crustal or mantle models.

In figure 4, horizontal phase velocities are estimated by sliding a $30 \mathrm{~s}$ time window along the time series of rotation rate and transverse acceleration and dividing peak amplitudes in time windows where the waveform fit is good (normalized maximum correlation coefficient greater than 0.95). The figure shows observed rotation rate and scaled transverse acceleration (top), theoretical rotation rate and scaled transverse acceleration (mid) and the phase velocity estimates (bottom) in a time window containing Love waves. Despite the difference in waveform and amplitude between synthetics and observations, the time-dependent phase velocities match surprisingly well the observations, supporting the conclusions from the analysis of another large event (Igel et al. 2005b). The Love wave phase velocity curve as a function of frequency is 

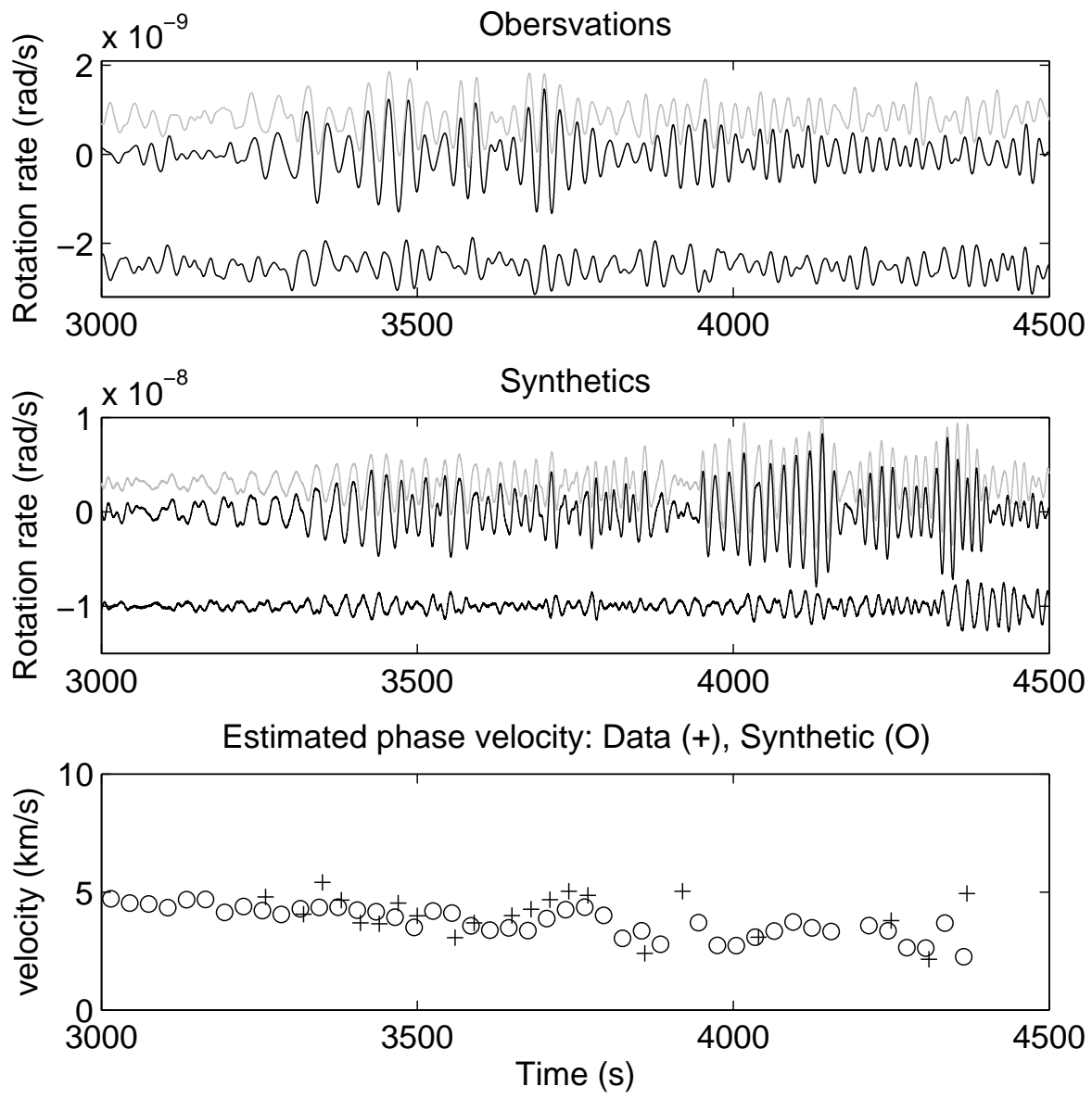

Figure 4: Estimation of phase velocities (lower plot shows velocities from observed data as crosses and velocities from simulation as circles) by correlation of observed rotation rate (gray) and transverse acceleration (black) in upper plots (the bottom trace is the residual). The chosen time window only displays part of the whole seismogram where mainly surface waves appear. See text for more details.

expected to reflect the material properties of some volume around the receiver location and may well be used for structural inversion if other information is not available. However, the uncertainties of this phase-velocity estimations still need to be investigated.

\subsection{Array based determination of rotational motion}

As explained in 3.1, all three components of rotation can in theory be derived from data acquired by an array of three-component seismometers at the Earth's surface. Indeed, in several array studies, rotation rate of seismic wavefields after large earthquakes was estimated (e.g., Huang 2003). However, there is only one study where array-derived rotation rate was compared with 


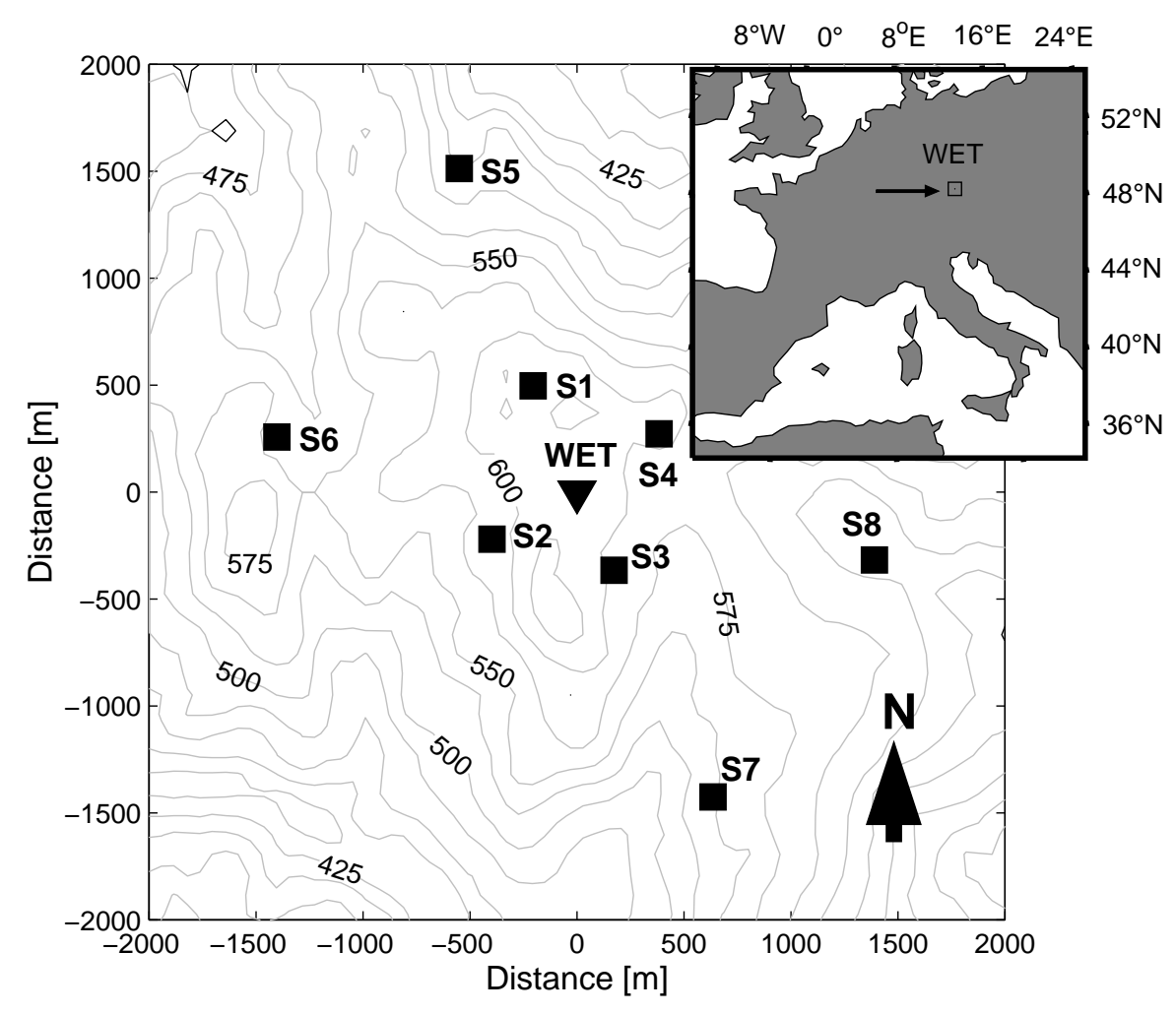

Figure 5: Topographic map of the Wettzell area, SE Germany. The Fundamental Wettzell Station (WET) is denoted by a triangle. The ring laser and the broadband GRSN seismometer are both located in WET (about $250 \mathrm{~m}$ away from each other).

direct measurements (Suryanto et al. 2005) and the results indicate that -as expected-array derived rotation is sensitive to noise. As indicated above and shown in Igel et al. (2005b), with appropriate scaling the broadband transverse acceleration can be almost identical to rotation rate provided that the assumption of plane wave propagation holds. Here we compare array derived rotation rate with transverse acceleration scaled with the appropriate phase velocity, to match the rotation rate amplitudes. This complements the studies of consistency between rotations and translations in phase and amplitude.

In December 2003 an array of seismometers was installed in Wettzell, about $150 \mathrm{~km}$ Northeast of Munich, Germany (figure 5) around the location of the ring laser. The radius of the array was about $1.5 \mathrm{~km}$. A broadband seismometer of the German Regional Seismic Network (GRSN) is collocated with the ring laser. The experiment ran up to early March 2004. The observational system consisted of 3 components of a Le3D-5s velocity sensor having a flat response in velocity between 0.2 and $40 \mathrm{~Hz}$, with a $400 \mathrm{~V} / \mathrm{m} / \mathrm{s}$ generator constant. The seismometers were either buried in soft ground at a bottom depth of about $50 \mathrm{~cm}$ or put on outcropping igneous rock boulders. The GRSN station is using a STS-2 instrument, with a flat response of 

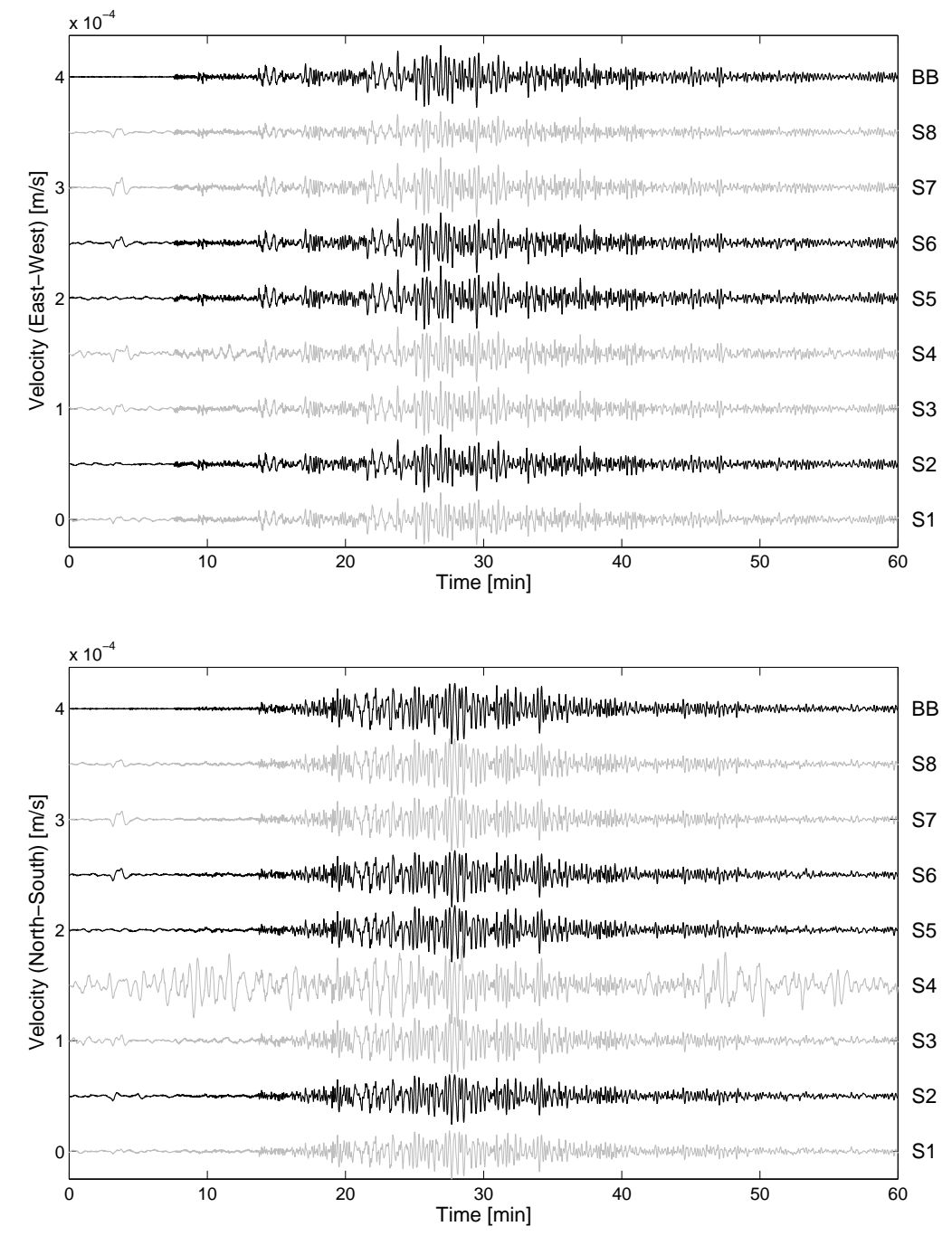

Figure 6: Horizontal components of the velocity recorded by the array seismometers for the December 26, 2003, Bam (Iran) earthquake. The data from stations 2, 5, 6 and broadband are used to derive the rotations. Top: East-West components; Bottom: North-South components.

the ground velocity from $8.33 \mathrm{mHz}(120 \mathrm{~s})$ to $50 \mathrm{~Hz}$.

Several teleseismic events with magnitude larger than 5.5 were observed during the experiment. Here we analyze one event: the M6.6 Bam (Iran) earthquake of December 26, 2003, with an estimated moment of $M_{0}=6.6 \times 10^{18} \mathrm{~N} . \mathrm{m}$ and an epicentral distance of $4425 \mathrm{~km}$. Rotations were determined as described in 3.1. In this study, we use four stations for both events to derive the rotation rates (figure 6).

In section 2.3 we have shown that, assuming a plane wave propagation, transverse horizontal acceleration and the vertical component of rotational rate should be in phase and their 

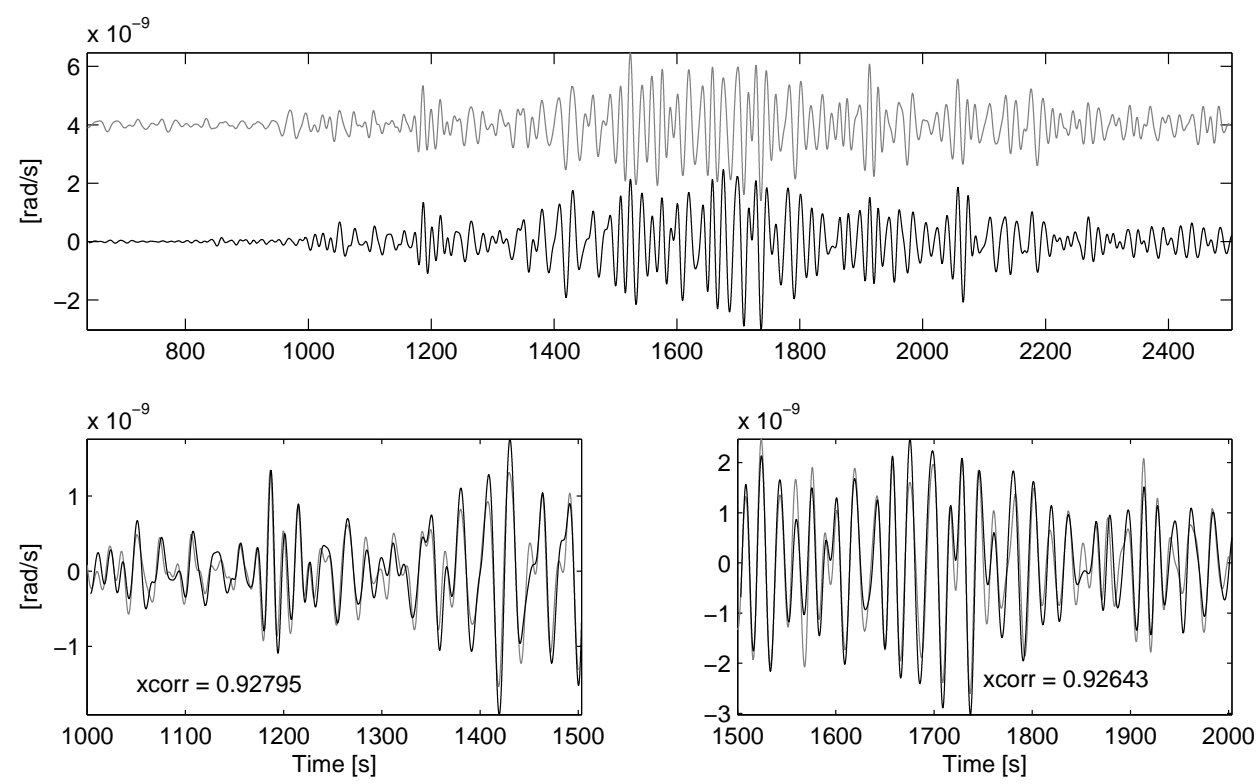

Figure 7: Comparison of collocated (scaled) transverse acceleration (black) observed by the GRSN broadband seismometer with array derived rotation rate (gray) for the Bam earthquake.

ratio equal to twice the horizontal phase velocity. Since the data from the ring laser was not available for this event, we use the transverse acceleration data to compare with the calculated array-derived rotational rate. This is justified given the substantial fit between rotation rate and transverse acceleration reported by Igel et al. (2005b) and Igel et al. (2005a) particularly for time windows containing the surface wave trains. For this purpose, a band pass filter $(0.03-$ $0.08 \mathrm{~Hz}$ ) is used to isolate the main phase of the surface waves contained in the seismograms. The transverse acceleration is scaled (i.e., converted to rotation rate) by dividing by twice a constant phase velocity $(5000 \mathrm{~m} / \mathrm{s})$. Figure 7 shows the computed time histories of the scaled transverse acceleration (black lines) superimposed with the vertical component of ground rotation rate (gray lines) across the array for the Bam earthquake. There is considerable phase match (maximum correlation coefficient above 0.9) between array-derived rotation rate and scaled transverse acceleration. This suggests and further supports the conclusion that the array seismograms, the broadband sensor and the ring laser (Suryanto et al. 2005) consistently provide the same information on the rotational part of the wave field.

\section{Discussion and Conclusions}

Are measurements of rotational ground motions useful for seismology and related fields in the Earth Sciences? While the tremendous success of research on the translational part of ground motions in the past decades would suggest that this is not the case, the question posed 
can only now be addressed in the context of real observations. The ring laser technology that was extended towards high resolution measurements of absolute rotations in the past years (Schreiber et al. 2003a, 2004) with the major aim of measuring changes of the Earth's rotation rate, was successfully transferred to the field of seismology, which required adaptation in terms of acquisition parameters and timing accuracy. The resolution of ring laser type measurements is now such that observations can be directly compared with those from broadband standard seismometers. In broadband global seismology we do not expect major information gain from rotation sensors as seismic network densities are ever increasing and many of the wavefield properties can be extracted by array processing. However -given the fact that we are dealing with a new observable- long period teleseismic broadband data provide an excellent opportunity to test the consistency of this new observable, thus the correct measurement of the ring laser instrument and its potential applicability in other domains of seismology (e.g., strong ground motion, source inversion, etc.).

As motivated in the theoretical section, assuming plane wave propagation, we can directly compare rotation rate with transverse acceleration as they should be in phase and related by a factor proportional to phase velocity. The first earthquake-induced observations with the German ring laser system (Igel et al. 2005b) demonstrate that indeed -particular for time windows containing the transversely polarized Love surface waves- the fit between those independent observations is almost perfect providing the test for phase consistency. The amplitude relation between acceleration and rotation rate can be used to estimate horizontal phase velocities as well as propagation directions. As shown in figure 4 these phase velocities match well those predicted by the most sophisticated forward modelling possible today. The accuracy and uncertainties of phase velocity estimates are still being investigated but it is important to note that -with collocated measurements of translation and rotation- it is thus possible to extract information from the wavefield that otherwise could only be obtained through seismic arrays. This may be useful in case of a very sparse network or when only single stations are possible, a situation likely in planetary seismology, but current technology would not yet allow a light sensor with the required sensitivity given the payload restrictions of space missions.

Sampling the seismic wavefield at the Earth's surface with an appropriate array allows the estimation of the rotational part by finite differencing the three components of ground motion for the given array geometry. While the first -surprisingly successful- direct comparison of ringlaser-based direct observation and array-derived rotational motions is given by Suryanto et al. (2005), it is clear that the logistic effort to determine rotations from array is considerably larger than direct measurements. This holds particularly for temporary arrays when the installation may be heterogeneous in terms of station quality. However, the fact that we are sampling the same rotation wavefield properties with the ring laser, broadband sensor and the array is further supported by the results shown in figure 7 . The rotation rate converted from the broadband transverse acceleration is close to the array-derived rotation rate as expected from theory.

We consider these studies as a contribution towards the demonstration of the accuracy and consistency of a new observable quantity in broadband seismology: the vertical component of rotation. While the acquisition technology will continue to improve there are still many open 
questions that need to be answered before it is clear what the new observable is good for. These questions involve (1) the sensitivity of rotations vs. translations with respect to Earth structure; (2) the importance of ground rotations in shaking hazard issues particularly in the presence of very unconsolidated low-seismic-velocity near surface structures; (3) the effects of strongly scattering structures and anisotropy; (4) the sensitivity of rotations with respect to finite fault scenarios; (5) the development of low-resolution portable sensors with the appropriate sensitivity for near source (e.g., aftershock) studies. Many of these issues can now be addressed using modern wave propagation simulation technology as was used in this study to model teleseismic observations.

\section{Acknowledgments}

This work was supported by the German Ministry of Research and Education (BMBFGeotechnologien). We also acknowledge support by the German Academic Exchange Service (IQN-Georisk), and by the KONWIHR project. We are grateful to J. Tromp and D. Komatitsch for providing their SEM code and to Ji Chen for the finite source parameters. We acknowledge the contributions of the Bundesamt für Kartographie und Geodäsie (BKG) towards the installation and operation of the $\mathrm{G}$ ring laser at the geodetic observatory Wettzell. Thanks to the Munich Leibniz Computing Center for providing access to their supercomputing facilities.

\section{References}

K. Aki and P. G. Richards. Quantitative Seismology. W. H. Freeman and Company, 1980.

K. Aki and P. G. Richards. Quantitative Seismology. University Science Books, $2^{\text {nd }}$ edition, 2002.

P. Bodin, J. Gomberg, S. K. Sing, and M. Santoyo. Dynamic deformations of shallow sediments in the valley of Mexico, part I: Three-dimensional strains and rotations recorded on a seismic array. Bull. Seismol. Soc. Am., 87:528-539, 1997.

M. Bouchon and K. Aki. Strain, tilt, and rotation associated with strong ground motion in the vicinity of earthquake faults. Bull. Seismol. Soc. Am., 72:1717-1738, 1982.

A. Castellani and Z. Zembaty. Comparison between earthquake spectra obtained by different experimental sources. Engng Struct., 18:597-603, 1996.

Ji Chen. 2004. URL http: //www.gps . caltech. edu/ jichen.

Cook. Concepts and Applications of Finite Element Analysis. John Wiley \& Sons, New York, 1974. 
J. Dyszlewicz. Micropolar theory of elasticity. Springer, 2004.

F. Galitzin. Vorlesungen über Seismometrie. B. G. Teubner, 1914. In german.

B.-S. Huang. Ground rotational motions of the 1999 Chi-Chi, Taiwan earthquake as inferred from dense array observations. Geophys. Res. Lett., 30:1307-1310, doi:10.1029/2002GL015157, 2003.

H. Igel, A. Flaws, A. Cochard, J. Wassermann, U. Schreiber, and A. Velikoseltsev. Rotational and translational motions induced by local, regional and global seismic events: I. observations and processing. 2005a. In preparation.

H. Igel, U. Schreiber, A. Flaws, B. Schuberth, A. Velikoseltsev, and A. Cochard. Rotational motions induced by the M8.1 Tokachi-oki earthquake, September 25, 2003. Geophys. Res. Lett., 32:L08309, doi:10.1029/2004GL022336, 2005 b.

C. Ji, D.J. Wald, and D.V. Helmberger. Source description of the 1999 Hector Mine, California earthquake; Part i. Bull. Seismol. Soc. Am., 92(4):1192-1207, 2002.

D. Komatitsch and J. Tromp. Spectral-element simulations of global seismic wave propagationI. Validation. Geophys. J. Int., 149:390-412, 2002a.

D. Komatitsch and J. Tromp. Spectral-element simulations of global seismic wave propagationII. 3-D models, oceans, rotation, and self-gravitation. Geophys. J. Int., 150:303-318, 2002b.

Dimitri Komatitsch. Méthodes spectrales et éléments spectraux pour l'équation de l'élastodynamique $2 D$ et $3 D$ en milieu hétérogène (Spectral and spectral-element methods for the $2 \mathrm{D}$ and $3 \mathrm{D}$ elastodynamics equations in heterogeneous media). $\mathrm{PhD}$ thesis, Institut de Physique du Globe, Paris, France, 1997.

Dimitri Komatitsch, Seiji Tsuboi, Chen Ji, and Jeroen Tromp. A 14.6 billion degrees of freedom, 5 teraflops, 2.5 terabyte earthquake simulation on the Earth Simulator. Proceedings of the ACM/IEEE Supercomputing SC'2003 conference, 2003. published on CD-ROM and at www.sc-conference.org/sc2003.

R. S. Lakes. Experimental methods for study of Cosserat elastic solids and other generalized continua. In H. Mühlhaus, editor, Continuum models for materials with micro-structure, chapter 1, pages 1-22. J. Wiley, 1995.

R. Teisseyre E. Majewski. Earthquake Thermodynamics \& Phase Transformation in the Earth's Interior. Academic Press, 2001.

G. A. Maugin. On the structure of the theory of polar elasticity. Phil. Trans. R. Soc., 356: 13671395, 1998. 
D. P. McLeod, G. E. Stedman, T. H. Webb, and U. Schreiber. Comparison of standard and ring laser rotational seismograms. Bull. Seismol. Soc. Am., 88:1495-1503, 1998.

R. L. Nigbor. Six-degree-of-freedom ground-motion measurement. Bull. Seismol. Soc. Am., 84: 1665-1669, 1994.

W. Nowacki. Theory of asymmetric elasticity. Pergamon Press, 1986.

A. Pancha, T. H. Webb, G. E. Stedman, D. P. McLeod, and K. U. Schreiber. Ring laser detection of rotations from teleseismic waves. Geophys. Res. Lett., 27:3553-3556, 2000.

K. U. Schreiber, T. Klügel, and G. E. Stedman. Earth tide and tilt detection by a ring laser gyroscope. J. Geophys. Res., 108:2132, 10.1029/2001JB000569, $2003 \mathrm{a}$.

K. U. Schreiber, A. Velikoseltsev, H. Igel, A. Cochard, A. Flaws, W. Drewitz, and F. Müller. The GEOsensor: A new instrument for seismology. In GEOTECHNOLOGIEN Science Report No. 3: "Observation of the System Earth from Space", Status Seminar, Programme \& Abstracts, Munich, 12-13 June 2003b. Bavarian State Mapping Agency (BLVA).

K. U. Schreiber, A. Velikoseltsev, M. Rothacher, T. Klügel, G. E. Stedman, and D. L. Wiltshire. Direct measurement of diurnal polar motion by ring laser gyroscopes. J. Geophys. Res., 109: B06405, 2004.

K. U. Schreiber, A. Velikoseltsev, G. E. Stedman, R. B. Hurst, and T. Klügel. New applications of very large ring lasers. In H. Sorg, editor, Symposium Gyro Technology, pages 8.0-8.7, $2003 c$.

B. Schuberth, M. Ewald, H. Igel, M. Treml, H. Wang, and G. Brietzke. To appear in: High Performance Computing in Science and Engineering, chapter Computational Seismology: Narrowing the Gap Between Theory and Observations. Springer, Heidelberg, 2005.

P. Spudich, L. K. Steck, M. Hellweg, J. B. Fletcher, and L. M. Baker. Transient stresses at Parkfield, California, produced by the $m$ 7.4 Landers earthquake of June 28, 1992: Observations from the UPSAR dense seismograph array. J. Geophys. Res., 100:675-690, 1995.

G. E. Stedman. Ring laser tests of fundamental physics and geophysics. Reports Progr. Phys., 60:615-688, 1997.

G. E. Stedman, Z. Li, and H. R. Bilger. Sideband analysis and seismic detection in a large ring laser. Appl. Opt., 34:7390-7396, 1995.

W. Suryanto, H. Igel, J. Wassermann, A. Cochard, B. Schubert, D. Vollmer, and F. Scherbaum. Comparison of seismic array-derived rotational motions with direct ring laser measurements. Bull. Seismol. Soc. Am., submitted, 2005. 
M. Takeo. Ground rotational motions recorded in near-source region of earthquakes. Geophys. Res. Lett., 25:789-792, 1998.

M. Takeo and H. M. Ito. What can be learned from rotational motions excited by earthquakes. Geophys. J. Int., 129:319-329, 1997.

R. Teisseyre, J. Suchcicki, K. P. Teisseyre, J. Wiszniowski, and P. Palangio. Seismic rotation waves: basic elements of theory and recording. Annali di Geofisica, 46:671-685, 2003.

M. D. Trifunac and M. I. Todorovska. A note on the useable dynamic range of accelerographs recording translation. Soil Dyn. and Earth. Eng., 21:275-286, 2001. 